\title{
Synthetic organisms and living machines
}

\section{Positioning the products of synthetic biology at the borderline between living and non-living matter}

\author{
Anna Deplazes $\cdot$ Markus Huppenbauer
}

Received: 27 April 2009/Revised: 12 June 2009/Accepted: 29 June 2009

(C) The Author(s) 2009. This article is published with open access at Springerlink.com

\begin{abstract}
The difference between a non-living machine such as a vacuum cleaner and a living organism as a lion seems to be obvious. The two types of entities differ in their material consistence, their origin, their development and their purpose. This apparently clear-cut borderline has previously been challenged by fictitious ideas of "artificial organism" and "living machines" as well as by progress in technology and breeding. The emergence of novel technologies such as artificial life, nanobiotechnology and synthetic biology are definitely blurring the boundary between our understanding of living and non-living matter. This essay discusses where, at the borderline between living and non-living matter, we can position the future products of synthetic biology that belong to the two hybrid entities "synthetic organisms" and "living machines" and how the approaching realization of such hybrid entities affects our understanding of organisms and machines. For this purpose we focus on the description of three different types of synthetic biology products and the aims assigned to their realization: (1) synthetic minimal cells aimed at by protocell synthetic biology, (2) chassis organisms strived for by synthetic genomics and (3) genetically engineered machines produced by bioengineering. We argue that in the case of synthetic biology the purpose is more decisive for the categorization of a product as an organism or a machine
\end{abstract}

\footnotetext{
A. Deplazes $(\bowtie)$

University Research Priority Programme (URPP) in Ethics, University of Zurich, Klosbachstr. 107, 8032 Zurich, Switzerland

e-mail: deplazes@ethik.uzh.ch

M. Huppenbauer

University Research Priority Programme (URPP) in Ethics, University of Zurich, Zollikerstrasse 117, 8008 Zurich, Switzerland
}

than its origin and development. This has certain ethical implications because the definition of an entity as machine seems to allow bypassing the discussion about the assignment and evaluation of instrumental and intrinsic values, which can be raised in the case of organisms.

Keywords Ethics - Life - Machines - Organisms · Philosophy $\cdot$ Synthetic biology $\cdot$ Synthetic life

\section{Introduction}

The difference between living and non-living matter is not only of great interest and challenge for science but also has philosophical and cultural impact. The question whether the living world is fundamentally different from the non-living world has been widely discussed in natural philosophy. Today the philosophical idea of vitalism, which says that the organic world is governed by different principles than non-living entities, is largely outdated. Nevertheless, even with the knowledge that living organisms follow the same chemical and physical laws as non-living matter but can develop novel properties by emergence (Luisi 2006, Chap. 6 ), it can be argued that there are differences for example between the traditional meaning of "living organism" and that of "machine". Living organisms traditionally exist, reproduce and change following natural rules, independently of the will of human beings. In contrast, in the artificial world human beings decide what exists, in what amount and in which form. A strict separation between the natural and the human-determined world has never existed, domesticated animals being natural and dependent on humans at the same time. However, living organisms designed, produced and fully controlled by human beings have been difficult to imagine until recently. Attempts to 
imitate or even create living organisms have been made since ancient times (Riskin 2007), but never led to something that would seriously be considered alive and yet not a member of the natural ${ }^{1}$ world. The rise of digital and biological technologies in the second half of the twentieth century has allowed for novel approaches to artificial forms of life. In vitro fertilization (IVF) allowed the fertilization of a human egg and thereby the production of a human embryo in the lab. Yet, the product of this procedure is still controlled by a natural design and is in that respect not more artificial than a naturally conceived human being. The progress in computer technology has lead to the development of disciplines called artificial life (AL) and artificial intelligence (AI). Human beings can produce digital "organisms" that reproduce, evolve and learn and thereby develop in unpredictable ways. They have life-like functions, but given that these "organisms" do not exist physically but only in a virtual world, they are so fundamentally different from natural life that there remains a clear boundary between natural life and life-like entities produced by computer technology. Synthetic biology adds a new chapter to the story of human-made life. Synthetic biology is an emerging technology at the interface between biotechnology, chemistry, engineering and computer science. Very different types of outputs, from genetically engineered bacteria to chemically synthesized genomes, to chemically assembled cells or even computer models of an artificial metabolism can all be considered intermediate- or end-products of synthetic biology. This multidisciplinary and multi-approach field has the unifying goal of producing and designing new forms of life (Deplazes 2009). In some respects the IVF and AL approaches are combined in synthetic biology. On the one hand, synthetic biologists use the basic natural mechanisms for their products, which means that they are trying to produce organic cellular structures controlled by a genome. On the other hand, their products should be regulated and controlled by a human design, similarly to computers. Depending on the approach, one or the other aspect (the usage of basic natural mechanisms as in IVF or the control by a human design as in AL) is more prominent. Interestingly, the aim of producing novel types of living organisms in synthetic biology not only implies the production of living from non-living matter, but also the

\footnotetext{
${ }^{1}$ In order to give a clear argument we define in this essay the term "natural" as a counterpart to "artificial", meaning independent of human plans and design. A natural process is thus one that proceeds independently of a human plan, a natural object is one whose design is independent of humans. A natural entity (belonging to the "natural world") can be subjected to artificial processes, as it is constantly the case for most human beings, and artificial entities do pass through natural processes as for example all of them are governed by natural laws.
}

idea of using living matter and turning it into machines, which are traditionally considered non-living.

It can be said that synthetic biology as a whole approaches the borderline between living and non-living matter from both sides, the living and the inanimate. In the following, this borderline will be examined and the position of different synthetic biology products in relation to this border will be discussed.

\section{Organisms and machines}

In order to investigate the borderline between the living and the non-living world in relation to the natural and artificial world we will in the following introduce and compare organisms and machines as representatives of natural and artificial entities. We are aware of the fact that the term machine is sometimes used in a metaphorical sense for natural objects ${ }^{2}$ and some machines such as certain robots are considered to be similar to living organisms. Furthermore, we are aware that previous technologies have already initiated the convergence of the two types of entities. However, for the sake of a transparent argumentation we use the two terms in their traditional meaning, which overlaps with the common usage as described by the definitions below, as discrete units and desist from using examples that can be considered as pre-stages of hybrid entities.

Also in this traditional meaning living organisms and machines have many common features. Both types of entities convert energy into mechanical forces. Many organisms and machines can move, all of them have an overall body-plan, which means that they are composed of different types of smaller subunits. Each single part of an organism or a machine has a different structure from the whole (in contrast to, e.g. a stone). Furthermore, organisms as well as machines follow a specific program.

\section{Machines}

According to the Advanced Learner's Oxford dictionary, a machine is "1. (often in compounds) an apparatus with several moving parts, designed to perform a particular task. Machines may be driven by electricity, steam, gas etc. or by human power [...]. 2. a group of people that control an organization, etc. or part of it [...] 3. A person who acts automatically without having to think or show any feelings" (Hornby 1995). The first part of this definition describes the common and generally accepted usage of this term (as we also use it in this article) according to

\footnotetext{
${ }^{2}$ This is for example the case when molecular- or cell biologists speak of the ribosomal machinery, degradation machinery, cell cycle machinery, etc.
} 
which machines fulfill a specific human purpose. Sewing machines are thus used for sewing, washing machines for washing, a CD player to listen to music, etc... In the second and third parts of the definition, "machine" is used in a metaphorical sense, which further clarifies its meaning. It points out that machines act automatically and mechanically and that there is no real independence or originality. Design and production of machines are normally established by human beings and machines exist because of and for the purpose of humans, they are artifacts. An "artificial machine" is thus a pleonasm because a non-artificial machine seems to be a contradiction; a machine is artificial per definition!

\section{Living organisms}

In the previously mentioned dictionary "organism" is defined as: "1. (a) a living being, esp a very small one, with parts that work together (b) an individual plant or animal 2. a system composed of parts which are dependent on each other" (Hornby 1995). The word "organism" originates from the same linguistic root as organize. (This meaning is reflected in the definition by the "parts that work together". Such an interaction can only function if the composition is well organized.) In this respect a machine could also be considered to be an organism. However, according to the first part of the definition organisms are alive. Organisms are living beings, and as all living beings fulfill the criteria suggested by the definition, namely to consist of parts that work together (for example organs or organelles), it can reciprocally also be said that all living beings are organisms. "Living organism" is thus a pleonasm, similarly to the artificial machine. ${ }^{3}$ The second part of the definition might extend the term to other systems besides living beings. Whether this second usage of the word, similarly to the case of the machine, is meant in a metaphorical sense or whether it should be considered a primary meaning of this notion depends on the interpretation. As mentioned above we are interpreting "organism" in this essay in its first meaning as a living entity.

Differences between the traditional concepts of organisms and machines

From the preceding descriptions, it is evident that there are important differences between machines and organisms.

\footnotetext{
3 Strictly speaking, "living organism" is not exactly the same type of pleonasm as "artificial machine", because "living" and "organism" have the same extension, every living entity is an organism and every organism is a living entity. This is not the case for "artificial machine", since "artificial" has a wider meaning than "machine", although every machine is indeed artificial most of the artificial entities are not machines.
}

We are comparing the two types of entities by consecutively examining four main features in which they differ. These four differences were determined by the compilation of the lexical understanding of the two terms and the biological definition of organism. ${ }^{4} \mathrm{We}$ are using them as a heuristic model to distinguish between the two types of entities. Although we consider them the four most fundamental differences, it is in principle possible that this list could be expanded by other properties. The four differences concern: (1) composition, (2) origin, (3) development and (4) purpose of the two types of entities.

First, concerning the composition, living organisms are cellular structures and each cell consists of organic material. ${ }^{5}$ They are regulated and controlled by a genome, which according to the central dogma of molecular biology is transcribed into RNA and translated into proteins. The composition is not merely relevant for the appearance of organisms but it is required or even responsible for most of their features such as heredity, growth, development or evolution. Machines, on the other hand, have up to now been built from inorganic material, be it metal, plastic or silicon. In contrast to the uniform structure amongst organisms, the structural composition of machines varies from one type to the other. The organization is not typical for all machines, as for instance the inside of a sewing machine has not much in common with that of a computer.

Second, the origin of organisms cannot be dated to a specific moment. They have evolved over millions of years by variation and selection and are still subject to these processes, the evolutionary transition from one species to another cannot be attributed to one moment. Machines, in contrast, are designed and produced by human beings and thus have a clearly defined origin. New types of machines arise when humans accomplish a novel design.

Third, and also related to their origins, the development of the two types of entities are different. Organisms develop, age and die, which implies programmed morphological and material changes. By contrast, machines do not develop or age in ways that involve programmed morphological and material transformations. They subsist in the form they have been built originally, without predicted changes during their existence. Furthermore, they are not actively involved in their own genesis or the continuity of their kind.

This feature is closely linked to the fourth difference between organisms and machines, namely their purposes. As mentioned before, both types of entities seem to follow a specific program. In case of the organism the genetic

\footnotetext{
4 Biological "definitions" of life refer to certain biological criteria such as metabolism, reproduction, growth, autopoiesis or evolution.

${ }^{5}$ We are here only considering living organisms as we know them from life on earth.
} 
program has developed through evolution optimizing the "fitness" of its carrier. It includes the abilities of selfproduction, self-organization and self-maintenance, which according to several authors, are most characteristic features of living organisms; they are summarized under the term "autopoiesis" (Luisi 2003). Autopoietic systems thus comprise or contain their own body plan and produce their own structures from organic material without direct external control or regulation. The fulfillment of the program, which implies survival and reproduction, thus does not serve any external purpose but is in the interest of the organism itself and its species. In contrast, the program of machines has been designed and written by human beings, and its fulfillment serves human purposes. Nothing can be in the interest of a machine but only in the interest of its owner who profits from it.

Given these differences between machines and organisms, the two types of entities seem to be largely dissimilar. Nevertheless, this article deals with two hybrid entities: artificial organisms and living machines, exchanging the adjectives of the two pleonasms "artificial machine" and "living organism"; it thus examines organisms with features of machines and vice versa. We first want to address traditional associations and expectations related to such hybrid entities, illustrated by some well-known examples from the science fiction literature.

\section{Artificial or synthetic organisms}

The terms "artificial" and "synthetic" do have closely related meanings. Both adjectives refer to something that has emerged by artificial synthesis. However, whereas "artificial" accentuates that the respective item is humanmade and thereby is opposed to "natural"; "synthetic" emphasizes that the item underwent a procedure of synthesis (implicating also that this is done by human beings). Both terms have been used in connection to "life", "organism" and "cell". As the term "artificial life" usually refers to other technologies more related to artificial intelligence than synthetic biology [although there are overlaps between AL and synthetic biology, particularly in the field of protocells (Bedau 2003)], we will generally use the term "synthetic" unless we specifically want to point at the contrast with "natural", in which case we will utilize the term "artificial".

A synthetic organism would thus be an organism that has been synthesized by human beings. It would, therefore, not fulfill all the classical features of an organism anymore. That said an entity that can grow, reproduce and develop without further human intervention could be considered an organism even when human beings have produced it. The first (the organic cellular composition) and the third (the development) of the four differences would still be characteristic for an organism, but not the second feature, the origin. According to such an understanding Frankenstein's creature would be a synthetic organism.

\section{Living machines}

This brings us to the second type of hybrid entities, the living machines. What would a living machine look like? According to the roboticist Rodney Brooks "living machines would be able to self-reproduce, find their own sources of energy, and repair themselves to some degree" (Brooks 2001). So far, nobody has succeeded in constructing such machines. If they existed, the origin of the first generation of these machines would remain humanmade and definable, also the composition could be that of classical machines. However, by being able of self-reproduction, finding the energy required for survival and selfreparation the machine would pursue its own interest. In order for a robot to be considered alive, it needs to be driven by its own interest and not by a human determined program. Descriptions of living robots from the science fiction genre illustrate this understanding of a living machine. An example is the robot wall-e, known from the film with the same name, which has been programmed to clean up human waste but falls in love with another robot.

These deliberations suggest that an ultimate synthetic organism might be an organism as characterized by its development, composition and purpose but not according to its origin, whereas a living machine would be a machine regarding its composition and its origin but not with respect to its purpose. Fictitious examples of hybrid entities such as Frankenstein or wall-e are challenging our ideas of a borderline between organisms and machines but by their fictitious character they are at the same time confirming the traditional categories, as they exist in the real world.

We will address next, how these two hybrid forms are interpreted in synthetic biology.

\section{Products of synthetic biology}

As outlined in the beginning of this article, synthetic biology is not a uniform discipline but an assembly of different approaches unified by a similar goal, namely the construction of new forms of life. In the following, three different approaches will be introduced without the aim of giving a complete picture of synthetic biology.

The protocell approach

According to R. V. Solé et al. the protocell approach, which they call "synthetic protocell biology" "aims at the 
construction of a chemical life-like ensemble in the form of an artificial cell system able to self-maintain, self-reproduce and potentially evolve" (Sole et al. 2007).

Ultimately such synthetic cells should have life-like properties. However, this does not mean that they should imitate life as we know it. So-called "minimal cells" or "protocells" could consist of the minimal numbers of genes (see also below) that would be transcribed and translated into the essential proteins. Alternatively, it is conceivable that a minimal cell would be a RNA-cell in which RNA would not only be the replicable carrier of genetic information but also fulfill catalytic functions as a ribozyme (Szostak et al. 2001). So far, self-maintaining, self-producing and evolvable protocells have not been realized, but different types of preliminary forms have been constructed or simulated. According to an overview on protocell research by Rasmussen et al., a protocell needs to integrate three main components, namely containment, metabolism and information (Rasmussen et al. 2009). For containment, vesicles are used, which similarly to the cell membrane, consist of a lipid bilayer. Metabolism includes some type of energy conversion that drives catalysis in the cell, and information is, for example, provided by a genome. Replicating vesicles or vesicles in which several types of biological reactions take place have been constructed, but several major challenges such as the coordination of different cellular processes, for example, in cell division, still need to be overcome before a real synthetic cell can be built. However, once this has been achieved, this would indeed be the production of a synthetic organism. Such a synthetic cell would fulfill the compositional and functional criteria of an organism but it would be created artificially. The production of such a cell would indeed be creation of life from non-living matter.

\section{Synthetic genomics approach}

Researchers of the synthetic genomics approach aim at producing a minimal organism based on a synthetic minimal genome, which is produced chemically. This genome would comprise only the absolutely essential genes that are required to survive under standardized lab conditions. Such a minimal genome should then serve as a "chassis-genome" that can be expanded by additional genes for specific functions that the organism is supposed to fulfill. In order to produce an organism that carries and expresses such a genome, a genome replacement methodology would be applied, in which the genome of an existing bacterium would be replaced by the synthetic chassis (or expanded chassis) genome. To date, such chassis organisms have not been produced. However, several of the single steps have been performed: A possible composition of the minimal genome has been proposed, the synthesis of a small bacterial genome has been achieved, and the principle of genome transfer from one bacterium to another has been established (Glass et al. 2006; Lartigue et al. 2007; Gibson et al. 2008). The three different procedures have not been combined yet, but the production of a chassis organism seems to be realizable along this line.

\section{Bioengineering approach}

Bioengineers aim at producing programmable bacteria or eukaryotic cells. They are designing elaborated biochemical pathways that they want to put into practice using a refined version of genetic engineering. The establishment of a library of different types of standardized genetic elements and other DNA fragments to control gene expression should facilitate the implementation of the designed pathways. In bioengineering, synthetic biology meets systems biology. The comprehensive view on organisms as living systems adopted by systems biology in order to explore the function of natural organisms is taken by synthetic biology in order to design for example an overall metabolism or regulatory system. Some synthetic biologists in this field like to refer to computers to explain their approach (Andrianantoandro et al. 2006). They want to design synthetic biology products analogously to the hierarchical structure of computers, which consist of modules with different gates carried out by physical layers. Bioengineers say that similarly, cells can be assembled from pathways of different biochemical reactions carried out by proteins. This comparison to computers seems to be more than a mere analogy between machines and organisms; rather, the bioengineered products should ultimately constitute real machines. Standardization, decoupling and abstraction should be introduced into biotechnology and result in biological systems with predictable behavior (Endy 2005). That these types of synthetic biology products are perceived as machines is also supported by the name given to the international student competition in synthetic biology iGEM, which stands for international Genetically Engineered Machine competition. The designing and fabricating aspects of these products define them as machines, but it would be hard to deny these entities are alive. We are thus confronted with a form of living machine.

The idea of bioengineered cells as a new type of machine has interestingly also been suggested in nanobiotechnology, where certain branches aim at producing cell-like robots. R. A. Freitas is known for his utopian models of nanorobots that behave like hyper-efficient erythrocytes, macrophages or other cells. In a review article on nanomedicine Freitas describes, under the subtitle of "biologic robots", certain "engineered bacterial 
Fig. 1 The position of synthetic biology products between organisms and machines. Arrows indicate the transition from wild organisms to machines designed for a specific function. The images stand for (1) wild organisms, (2) domesticated organisms, (3) genetically modified organisms, (4) synthetic cells, (5) chassis organisms, (6) genetically engineered machines, (7) intelligent machines, $(8)$ computers, (9) machines with one specific function
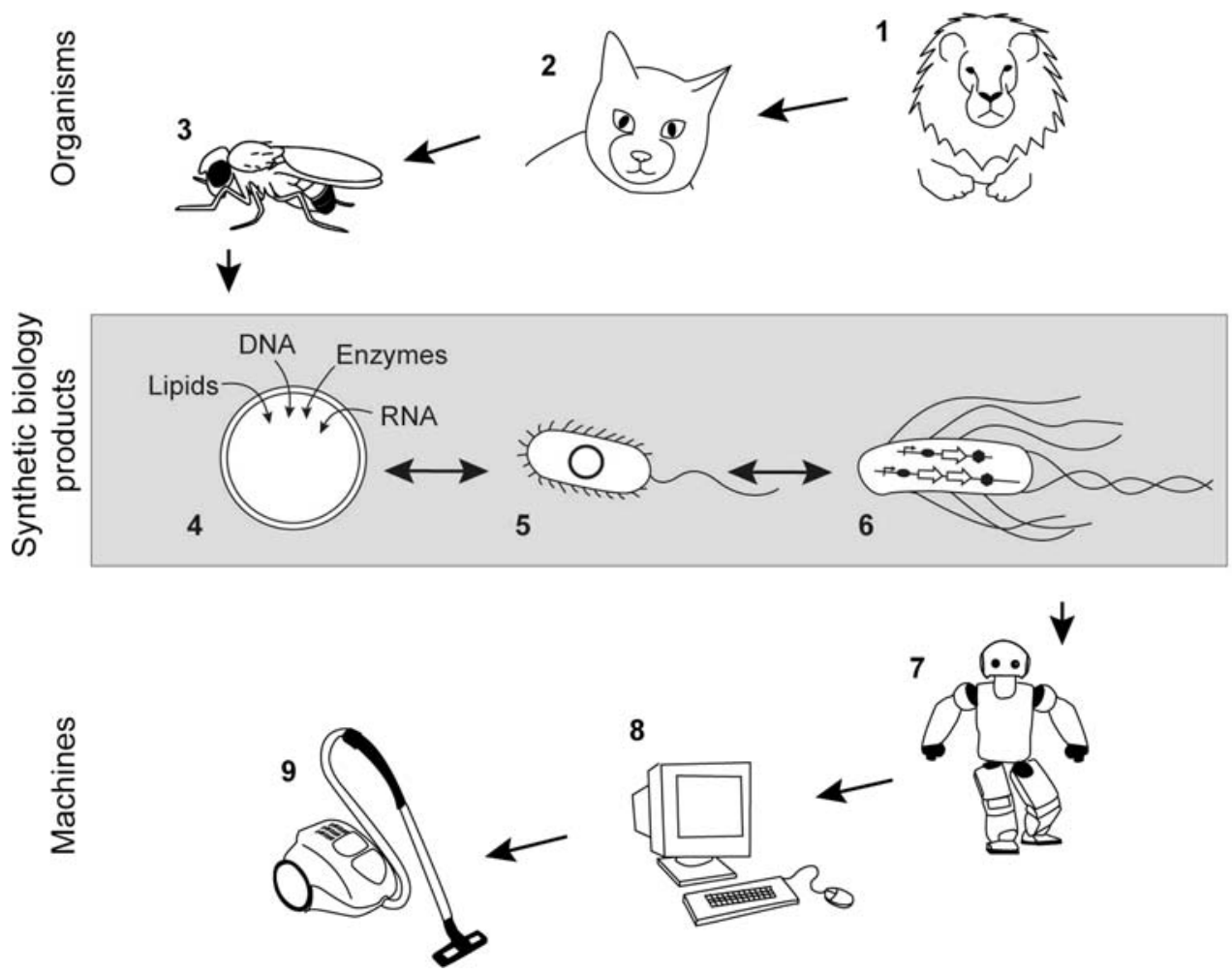

8
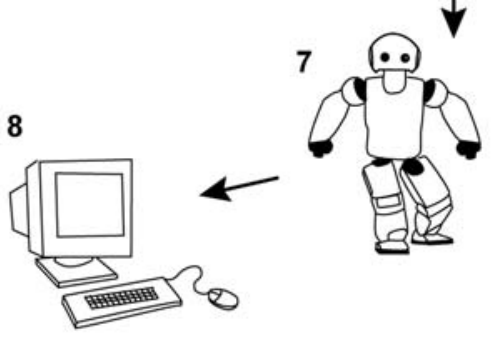

biorobots", which he also refers to as synthetic microbes designed to perform medical functions ${ }^{6}$ (Freitas 2005).

Comparing the products of the different synthetic biology approaches

Considering the three described synthetic biology products at the interface between living and non-living matter, one may wonder where these objects would be integrated in a diagram of increasingly machine-like entities as depicted in Fig. 1.

Synthetic cells would be composed of the same type of molecules as natural organisms. However, their origin would be fully artificial. Since these cells should be able to maintain themselves, reproduce and evolve, their development would be that of natural organisms in spite of their artificial origin. Particularly interesting in this case is the capability of those entities to evolve, which raises the question whether in subsequent generations, at a point when new structures and features have evolved, their origin should still be considered artificial or whether the "processing by evolution" would render them natural. Similarly to natural cells, protocells in basic research would try to

\footnotetext{
6 The biorobots of R. A. Freitas are imagined products in which aims of synthetic genomics are combined with bioengineering. This example should not describe synthetic biology but merely illustrate how synthetic organisms can be conceived as a hybrid between organism and machine.
}

secure their own survival and reproduction. However, later versions of such cells would also be designed in order to serve human purposes (Pohorille and Deamer 2002). Taken together, composition and development of these entities would be organism-like whereas their origin would be machine-like. At least in basic research they would not be designed to fulfill purposes for human beings and would thus be comparable with living organisms.

Chassis organisms with a synthetic genome would also consist of an organism-like composition and development since they should be able to maintain themselves and reproduce. Although the origin of their genomes would be artificial, the origin of the chassis organism would not be fully artificial because the synthetic genome would be introduced into a natural cell. The chassis-function of such a minimal organism implies that the minimal genome should be expanded by genes required to fulfill certain functions. This human-determined purpose would correspond with that of a basic machine.

The products of bioengineering consist of the same biological components as natural organisms. Also, their development is largely similar to that of organisms. However, ultimately they should be deterred from evolving and performing functions that are not required for their designed purposes. Therefore, the natural development of these entities would be intentionally impaired. Whereas the design of these organisms is clearly artificial, their material origin is mainly natural as cytoplasm and genome of the 
original cell are still required. The most machine-like feature of bioengineered cells is their purpose, which ideally would be completely defined by humans.

The summary of this comparison in Table 1 indicates that for products of synthetic biology the features that traditionally are used to distinguish between machines and organisms are not correlating anymore. On the one hand, a synthetic cell can be like a machine concerning its origin but almost like a living organism according to its development and purpose. A bioengineering product, on the other hand, should functionally be like a machine but its origin and development are still very close to that of traditional organisms. Synthetic biologists perceive the synthetic cell as a synthetic organism whereas the bioengineering product is described as a living machine. Interestingly, this different perception of the two products does not reflect the assignment of machine-like or organism-like properties as presented in Table 1, where both entities have the same number of + (machine-like features) and almost the same number of - (organism-like features). This discrepancy illustrates that the actual naming by synthetic biologists "living machine" or "synthetic organism" does not reflect the occurrence of machine-like or organism-like features as represented by the table. Not only are characteristics of machines and organisms mixed, but also an entity with more organism-like features is not necessarily perceived as an organism (as illustrated by the prevalence of organism-like features for the bioengineering product). The features that according to a traditional understanding of the two types of entities were decisive for the perception of an entity as a machine or an organism, respectively, do not have this function anymore in case of the assignment of synthetic biology products. The clear-cut difference of what is perceived as a machine and what is perceived as an organism is being blurred; hybrid entities seem to become reality.

\section{Implications of the nomenclature}

Although the names given to synthetic biology products do not inform us about the resemblance of the respective entity to machines and organisms they are meaningful. They reveal something about the objectives synthetic biologists are striving for with the respective product as well as the position and status that can or should be assigned to it. Furthermore, the nomenclature will certainly influence the public perception of synthetic biology products because it raises certain expectations and worries.

\section{Aims of synthetic biologists}

Discussing products of synthetic biology so far means discussing aims of synthetic biologists because the described versions of the respective products have not been realized yet. To date there are neither fully controllable bioengineered bacteria nor completely synthetic cells nor minimal organisms carrying chassis genomes. Therefore, it is particularly interesting to look at the names of future synthetic biology products in respect to what they reveal about their implication rather than about their physical consistence. Already the definition of "machine" and "organism" that we have considered above comprised metaphorical applications of the two terms, suggesting that these terms evoke certain associations. By calling their product an artificial or synthetic cell, scientists are announcing that life no longer is only a natural process and feature. They point out that the phenomenon "life" will be fully understood by scientists, and this understanding should enable the production of life. They thereby position their product next to living organisms in spite of its artificiality. This distinguishes the achievement of producing such an entity from all the other artificial objects.

Table 1 How products of synthetic biology are blurring the borderline of what we perceive as machines and organisms

\begin{tabular}{|c|c|c|c|c|c|}
\hline & Machine & $\begin{array}{l}\text { Bioengineering } \\
\text { product }\end{array}$ & $\begin{array}{l}\text { Synthetic genomics } \\
\text { product }\end{array}$ & $\begin{array}{l}\text { Synthetic } \\
\text { cell }\end{array}$ & Organism \\
\hline Material & ++ & -- & -- & -- & -- \\
\hline Origin & ++ & - & -+ & ++ & -- \\
\hline Development & ++ & - & -- & -- & -- \\
\hline Purpose & ++ & ++ & + & - & -- \\
\hline
\end{tabular}

The table illustrates how similar the three synthetic biology products are compared to machines concerning the four categories: material, origin, development and purpose. ++ indicates that the feature is machine-like; -- stands for an organism-like feature; + indicates that the respective feature is not exactly as in machines, but more similar to a machine than to a natural organism; - indicates that the respective feature is more similar to a natural organism than to a machine; -+ indicates that the feature is about as much machine-like as organism-like. The assignment of + and - values is largely generalized and there are certainly exceptions that tend to loose one or the other feature, this is particularly true for the "Machine" and "Organism" reference-categories. However, the detachment from the original feature is never as pronounced as in case of the synthetic biology products 
Calling a genetically engineered bacterium a machine conveys a completely different message. It points out that these entities are controlled and produced by human beings who can dispose of them freely and that these entities are no longer part of the realm of nature.

Naming synthetic biology products

Analyzing which synthetic biology products are presented as living machines and which of them as synthetic organisms, it seems that the noun is determined by the purpose of the entity, whereas the descriptive adjective represents the origin.

The living machines should fulfill human purposes, which is typical for machines but have the natural origin characteristic for organisms. Synthetic cells in basic research would be considered real synthetic organisms if they are autopoietic, reproduce and evolve. In other words similarly to natural organisms, they would promote their own survival and reproduction, but their emergence and origin would be artificial. This emphasis in the nomenclature is not compulsory. Theoretically, it would also be possible to call a bioengineered bacterium a synthetic organism or a protocell a living machine. The observation that the first version of nomenclature is applied tells us something about the importance of the different features of organisms and machines. Since the noun is decisive for the categorization of the entity, the importance of the purpose of the entity seems to outweigh its origin. This might indicate a new emphasis in defining living organisms and life in general, which is in contrast to the fictitious idea of living machines as described at the beginning of this article. The wall-e robot would ideally be similar to machines concerning his origin and living concerning his purpose.

Position and status of the product and its relation to nature

The focus on the purpose of synthetic biology products in order to assign them either to organisms or machines is also interesting concerning its moral status. There are various theories on how living organism should be considered morally. Mostly, the discussion involves the question what kind of values can be assigned to organisms. Some commentators argue living organisms have intrinsic value, meaning that they are regarded as valuable in and of themselves, independently of their usefulness for human beings. Other positions state that at least lower forms of living organisms do not have intrinsic but only instrumental value, which depends on their usefulness for human beings. If the value of an organism is mainly set to be instrumental this implies that the organism can be used for human purposes. However, for positions arguing for an intrinsic value of organisms, their instrumentalization needs to be well justified in order to be morally acceptable (O'Neill 2003). The purpose-oriented perception of entities in synthetic biology avoids this discussion by defining entities as machines, as soon as the aspect of their function for human purposes predominates. No one denies that it is justified to instrumentalize machines, as it is part of their definition that they perform a particular task for human beings.

\section{Public perception}

Because of the traditionally clear-cut differences between organisms and machines, the public perception of a product will depend on how it is called and propagated. The nomenclature of synthetic biology products thus might be a factor promoting public reactions. As mentioned in the beginning there are certain ideas and imaginations related to the terms "synthetic organisms" and "living machines". Versions of the two terms such as "genetically engineered machines" or "artificial cells" as well as the name of the full discipline "synthetic biology" are likely to raise certain associations.

\section{Conclusion}

This comparison of organisms and machines and the position of synthetic biology products with respect to these two types of entities has illustrated that the borderline between the two is continuously becoming blurred. This is not only happening because of the metaphorical usage of the terms (as indicated already in the definitions in the beginning) and because of the imagination of hybrid entities but also because in novel entities such as products of synthetic biology, features that used to be characteristic for one group are combined with features of the other type of entity. Synthetic cells with an artificial origin should eventually have the composition and development and, in some cases, the functions of organisms. Products of bioengineering, which are derived from living beings, should follow the functionality and controlled development of machines. The two groups "machines" and "organism" that we have treated as discrete classes in this essay have been stretched to a lower extent by other technologies such as breeding or genetic engineering. But with synthetic biology novel entities seem not to stretch but to transgress the borderline between organisms and machines, the nomenclature of the synthetic biology products reflects this transition.

The idea that there might be hybrids combining features of machines and organisms is not new, but the notion of how such beings would look like and how they would be 
evaluated, is changing in the light of synthetic biology. Synthetic organisms are not imagined as copies of human beings but as new, minimal forms of life. Living machines in synthetic biology are not imagined as mechanical beings but as organisms that are fully controlled by human beings. The difference in our conception of the two types of entities therefore gets more blurred. These new forms of life will affect the concept and evaluation of life and the idea of what constitutes a machine in society and in our culture. In some decades the definitions of "machine" and "organism" in the mentioned dictionary might be expanded by their usage in synthetic biology. This technology might provide a new example how science influences culture. It will be necessary to separate different aspects of our understanding of life. In particular, ideas related to a "self of living organisms", an "intrinsic value" or an "inviolability of life" might not necessarily be appropriate for simple forms of synthetic organisms. However, if and when higher forms of life are discussed, the moral meaning of their "self-interest" might have to be revisited and contrasted to machines. It thus seems that synthetic biology will give us plenty of opportunity to discuss and adapt our concept of life in the coming decades, and will be a chance to refine our biological and philosophical understanding of this phenomenon.

Acknowledgements We thank Agomoni Ganguli-Mitra for critical reading of the manuscript and valuable comments. This work was supported by the University Research Priority Programme (URPP) Ethics of the University of Zürich.

Open Access This article is distributed under the terms of the Creative Commons Attribution Noncommercial License which permits any noncommercial use, distribution, and reproduction in any medium, provided the original author(s) and source are credited.

\section{References}

Andrianantoandro E, Basu S, Karig DK, Weiss R (2006) Synthetic biology: new engineering rules for an emerging discipline. Mol Syst Biol 2:1-14
Bedau MA (2003) Artificial life: organization, adaptation and complexity from the bottom up. Trends Cogn Sci 7:505-512

Brooks RA (2001) Steps towards living machines. In: Gomi T (ed) Evolutionary robotics from intelligent robotics to artificial life. Springer, Berlin, pp 72-93

Deplazes A (2009) Synthetic biology-piecing a puzzle together. EMBO Rep (forthcoming)

Endy D (2005) Foundations for engineering biology. Nature 438: 449-453

Freitas RA Jr (2005) What is nanomedicine? Nanomedicine 1:2-9

Gibson DG, Benders GA, Andrews-Pfannkoch C, Denisova EA, Baden-Tillson J, Zaveri H, Stockwell TB, Brownley A, Thomas DW, Algire MA, Merryman C, Young L, Noskov VN, Glass JI, Venter JC, Hutchison CA III, Smith HO (2008) Complete chemical synthesis, assembly, and cloning of a Mycoplasma genitalium genome. Science (New York, N.Y.) 319:1215-1220

Glass JI, Assad-Garcia N, Alperovich N, Yooseph S, Lewis MR, Maruf M, Hutchison CA III, Smith HO, Venter JC (2006) Essential genes of a minimal bacterium. Proc Natl Acad Sci USA 103:425-430

Hornby AS (1995) Oxford advanced learner's dictionary. Oxford University Press, Oxford

Lartigue C, Glass JI, Alperovich N, Pieper R, Parmar PP, Hutchison CA III, Smith HO, Venter JC (2007) Genome transplantation in bacteria: changing one species to another. Science (New York, N.Y.) $317: 632-638$

Luisi PL (2003) Autopoiesis: a review and a reappraisal. Die Naturwissenschaften 90:49-59

Luisi PL (2006) The emergence of life, from chemical origins to synthetic biology. Cambridge University Press, Cambridge

O'Neill J (2003) The varieties of intrinsic value. In: Light A, Rolston H III (eds) Environmental ethics. Blackwell Publishing, Malden, pp 131-142

Pohorille A, Deamer D (2002) Artificial cells: prospects for biotechnology. Trends Biotechnol 20:123-128

Rasmussen S, Bedau MA, Chen L, Deamer D, Krakauer DC, Packard NH, Stadler PF (2009) Introduction. In: Rasmussen S, Bedau MA, Chen L, Deamer D, Krakauer DC, Packard NH, Stadler PF (eds) Protocells, bridging nonliving and living matter. The MIT Press, Cambridge

Riskin J (2007) Introduction: the Sistine gap. In: Riskin J (ed) Genesis redux, essays in the history and philosophy of artificial life. University of Chicago Press, Chicago, pp 1-34

Sole RV, Munteanu A, Rodriguez-Caso C, Macia J (2007) Synthetic protocell biology: from reproduction to computation. Philos Trans R Soc Lond 362:1727-1739

Szostak JW, Bartel DP, Luisi PL (2001) Synthesizing life. Nature 409:387-390 